\title{
AUTOMATION TOOLS FOR ENHANCING GROUND-OPERATION SITUATION AWARENESS AND FLOW EFFICIENCY
}

\author{
Victor H. L. Cheng, AIAA Associate Fellow \\ Optimal Synthesis Inc. \\ Los Altos, California
}

\begin{abstract}
In view of the ever-increasing air traffic, much attention in air-traffic-management research has been given to improving arrival and departure efficiency. As air traffic begins and ends at the airport, the issues of taxi delays and ground-operation incursions become more pressing. This paper considers the surface-traffic problem at major airports and envisions a collaborative traffic and aircraft control environment where a surface traffic automation system will help coordinate surface traffic movements. A previous study has established the performance potential of advanced guidance and control of a transport aircraft to deliver high-precision taxi capability. Such an aircraft capability will provide the surface traffic automation system with the flexibility to issue taxi clearances with tight time margins, in an effort to reduce taxi delays. An example is the ability to clear taxiing aircraft to cross active runways within a precise time window, without the need for the aircraft to hold short of the runways and unnecessarily increase taxi delay while waiting for the opportunity to cross. This paper describes the development of a surface traffic automation system, known as Ground-Operation Situation Awareness and Flow Efficiency (GO-SAFE). The GO-SAFE system is designed to work with advanced surveillance and communications technologies, and anticipated air traffic automation systems under development. It includes tools to help the ground controller with predicted traffic information, functionality to manipulate taxi routes, and advanced capabilities to schedule runway usage to accommodate landing, takeoff, and crossing traffic. Some of the GO-SAFE functionality will be useful for current-day operations, and its advanced tools will provide maximum benefit when integrated with advanced aircraft taxi control capabilities.
\end{abstract}

\section{INTRODUCTION}

The anticipated increase in air travel demands a more efficient air transportation system to handle the increased traffic. Government agencies including the National Aeronautics and Space Administration (NASA) and the Federal Aviation Administration (FAA) are researching advanced technologies to

\author{
and \\ David C. Foyle \\ NASA Ames Research Center \\ Moffett Field, California
}

provide the efficiency enhancements. Ref. 1 contains a brief description of such programs: Center-TRACON Automation System (CTAS) ${ }^{2}$, with automation aids for the Air Route Traffic Control Center (ARTCC or Center) and the Terminal Radar Approach Control $(\text { TRACON })^{3}$; Terminal Area Productivity (TAP) $\operatorname{program}^{4-9}$; Surface Movement Advisor (SMA) ${ }^{10,11}$; and Advanced Air Transportation Technologies (AATT) program. Current experience with CTAS has been extremely successful in fulfilling its objectives of enhancing traffic efficiency through time-based metering. As it improves the efficiency in arrival traffic, airport surface traffic will become a weak link in the air-traffic equation if it is not accorded the attention commensurate with other air traffic automation tools.

The air transportation system often has to contend with two seemingly opposing issues: total throughput and safety. To increase total throughput, it is often necessary to increase the number of runways and taxiways for handling the increased traffic. Examples of such growth include the expansion of the Dallas/Fort Worth International Airport (DFW) from the previous six-runway configuration to the proposed eight-runway metroplex, and the recent replacement of the Denver Stapleton Airport with the much larger Denver International Airport (DEN). DFW is in the middle of the expansion effort and currently has seven runways, with Figure 1 labeling in parentheses the proposed changes in runway layout due to the addition of the eighth runway. Such expansion generally will also increase the complexity of the airport configuration. Under most airport configurations, adding runways results in some runways blocking the traffic between the terminal ramp area and other runways further out. As the tower controllers have more flights to control, they also have more taxiway intersections and runway crossings to worry about. If the increase in traffic leads to operational changes to reduce aircraft separation for increasing efficiency, the increased throughput of the outer runways will lead to a further increase in the need for runway crossings. Furthermore, a similar increase in throughput of the inner runways reduces the opportunity for runway crossings to take place. These operational changes to accommodate the increasing traffic compound the safety and efficiency issues. 


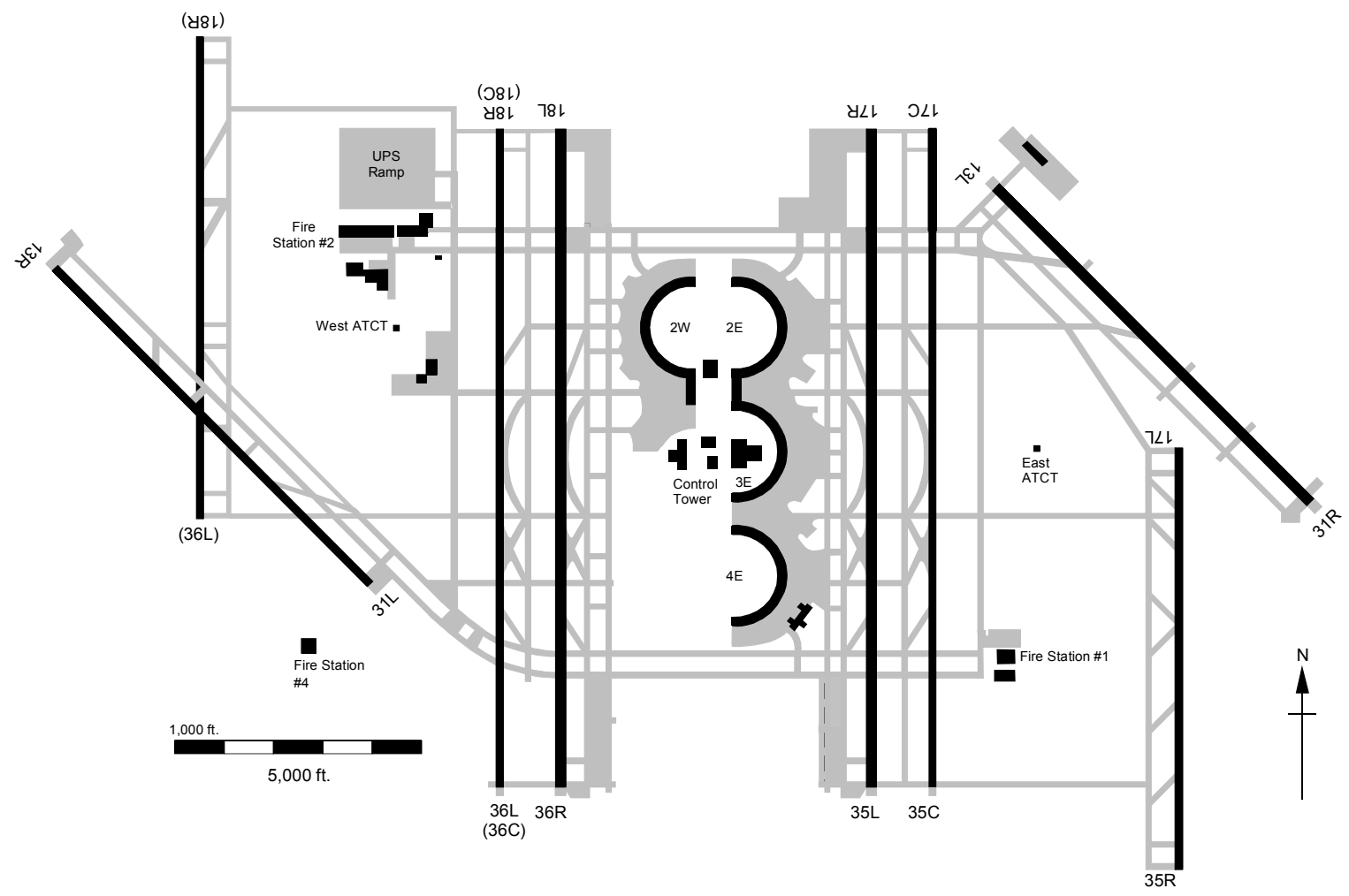

Figure 1. Layout of Dallas/Ft. Worth International Airport (DFW)

For airports such as DFW with complex runway configurations to deliver high throughput with hub-andspoke flight operations, runway-crossing delay is a major efficiency issue that has been well documented ${ }^{12}$. Current south-flow operations at DFW, which account for the majority of the operations at this airport, use Runway $17 \mathrm{R}$ for departure and $17 \mathrm{C}$ for arrival. During rush periods, the arrival flights on $17 \mathrm{C}$ often have to queue up at the three taxiways EL, EM and B (see Figure 2) after exiting from M3, M5 and M6, respectively, before they are cleared to cross $17 \mathrm{R}$ together as a group. Such holding prior to activerunway crossing means that sometimes three flights would line up for each of the three taxiways, a total of nine flights, before they are allowed to cross. This introduces substantial taxi delay to most of these flights. As a result, to achieve proportionate increase in throughput from addition of new runways and taxiways, the surface operation often needs to resort to tactics such as grouping flights from runway crossing to minimize disruption of landing and takeoff, at the expense of increasing total taxi delay.

As pointed out in Ref. 1, results from an MIT study ${ }^{13}$ are consistent with the notion that the taxiing traffic requiring active-runway crossings experiences substantial taxi-delays when the runways are heavily occupied by takeoff and landing traffic. Reference 14 indicates that, for departure traffic, there would be substantial savings by converting runway queuing time into gate delays. It is therefore reasonable to conclude that minimization of unnecessary taxi time would increase savings for both departure and arrival traffic, even if it means more gate holding delays. Gate holding schemes such as those studied in reference 14 can be used in conjunction with a surface traffic automation system that controls the taxiing traffic.

As for safety, a very serious surface-traffic safety issue is the runway incursion problem, which is being addressed by major programs sanctioned by the FAA and the International Civil Aviation Organization (ICAO). The FAA Runway Incursion Reduction Program (RIRP) ${ }^{15}$ studies technologies that can provide improved surveillance information to enhance situation awareness of air traffic control (ATC) and the flight crew. Technologies being evaluated by RIRP include the Airport Target Identification System (ATIDS) ${ }^{16}$, Airport Surface Detection Equipment (ASDE-3 and ASDE-X) $)^{17}$, Inductive Loop Technology ${ }^{18}$, Automatic 


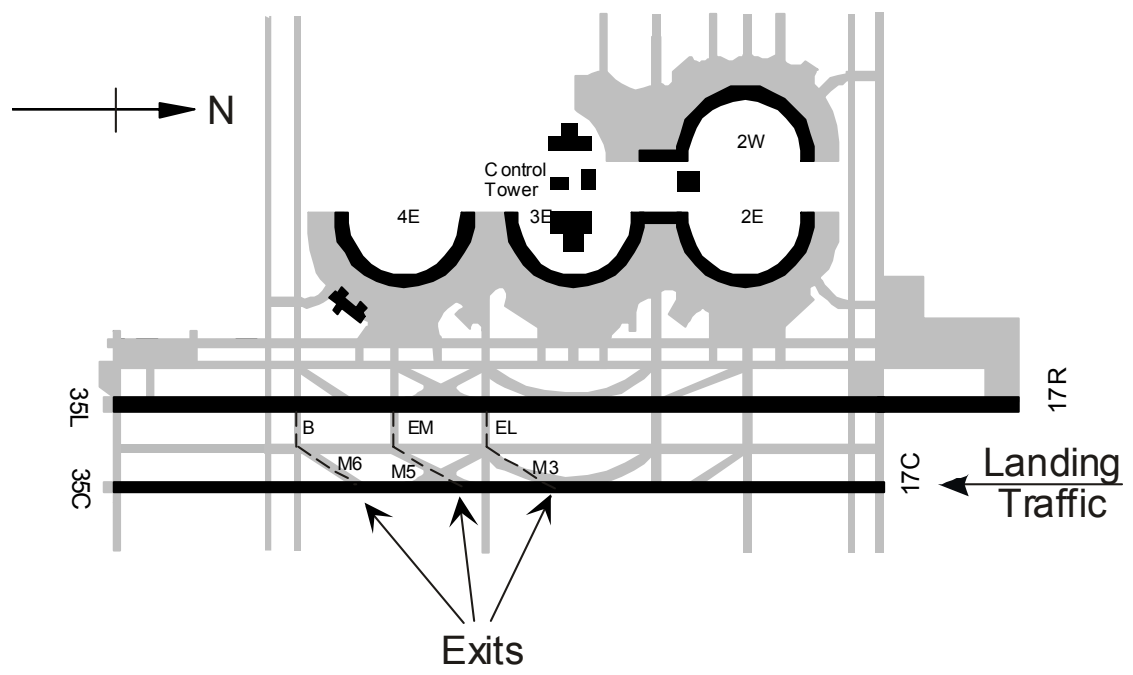

Figure 2. Example of Landing, Turn Off and Runway Crossing at DFW surveillance sources directly into the vehicle control system to enhance situation awareness for incursion avoidance. In the near term, the far-term ideas need to be adapted to address limited datalink functionality, limited surveillance technologies, and manual pilot control.

This paper describes the development effort of a surface traffic control automation system. The system, known as GroundOperation Situation Awareness and Flow Efficiency (GO-SAFE), is composed of several tools for improving the ground controller's awareness of the traffic situation and for providing advisories to Dependent Surveillance - Broadcast (ADS-B) ${ }^{19}$, and the Surface Surveillance Data Server. The ICAO Advanced Surface Movement Guidance \& Control System $(\mathrm{A}-\mathrm{SMGCS})^{20}$ is another concept which includes features and functions to enable safe and efficient airport surface operations. As these major programs focus heavily on the safety issues of surface traffic, the current study explores the use of automation technologies for improving surface traffic efficiency, with the assumption that many of the communication, navigation and surveillance (CNS) technologies being studied by these major programs will be available.

The study reported in this paper is part of a concept to achieve collaborative traffic and aircraft control for improving efficiency while maintaining or enhancing safety in airport surface operations. A previous study has established the potential use of state-of-the-art automatic control technologies to enable highprecision taxi ${ }^{1}$, delivering taxi control performance well within the requirements specified for A-SMGCS. The envisioned collaborative environment includes a surface traffic control automation system for coordinating traffic in a more orderly manner, including the possibility to allow flights to execute active-runway crossing under tightly controlled conditions. In the far term, the system may involve ground clearances including complete optimal taxi routes with specific time markers issued via data link. In addition, auto-taxi may be possible, and nearby vehicle traffic data can be automatically fed from and architecture of GO-SAFE is discussed in the next section, followed by descriptions of its three tool components in subsequent sections. The last section concludes the paper with a summary and remarks.

\section{GROUND-OPERATION SITUATION AWARENESS AND FLOW EFFICIENCY (GO-SAFE) SYSTEM CONCEPT}

It is common to perceive air traffic operations as a controlling air traffic by simply reducing the required separation between flights can increase air traffic efficiency at the cost of reduced safety (Figure 3). Use

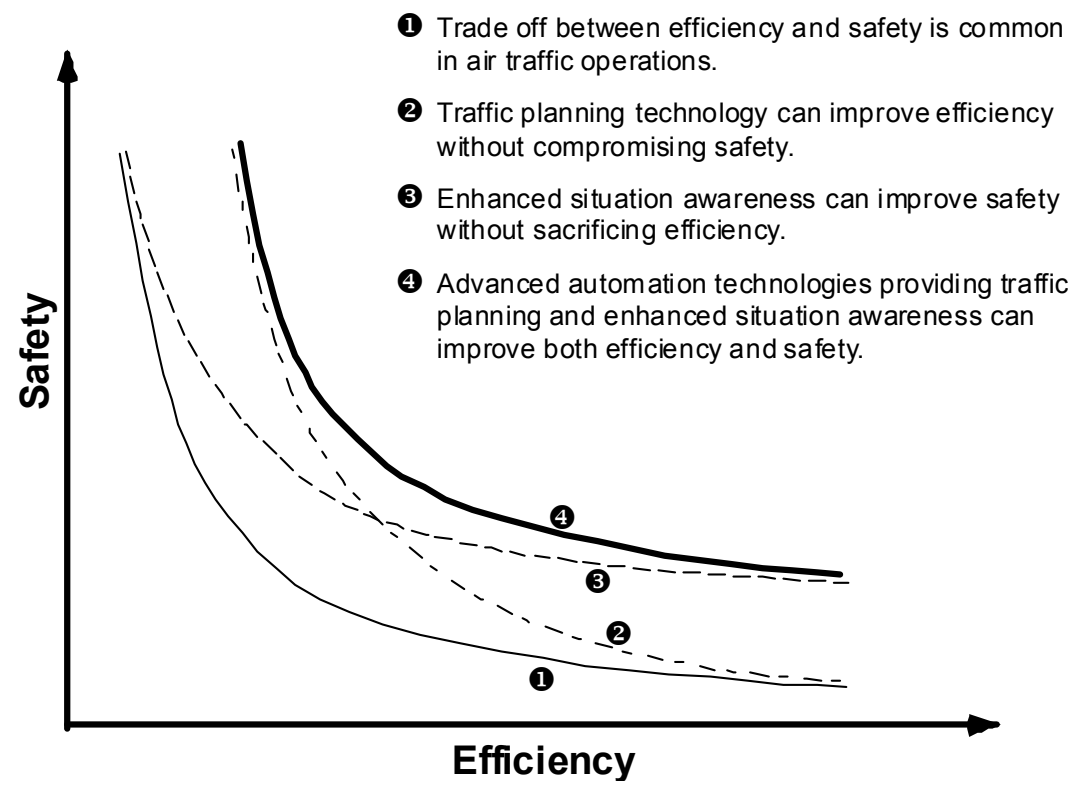

Figure 3. Traditional Tradeoff between Efficiency and Safety enhance flow efficiency. The overall system concept tradeoff between efficiency and safety. For instance, 
of traffic planning technology, such as CTAS for improving sequencing and scheduling, can improve efficiency without compromising safety. On the other hand, use of technologies to improve the controller's awareness of the air traffic situations can improve safety without sacrificing efficiency. Through appropriate use of advanced technologies, these beneficial attributes can be realized and combined.

For surface traffic control, the Ground-Operation Situation Awareness and Flow Efficiency (GO-SAFE) environment consists of three tools to improve both efficiency and safety: the Ground-Operation Prediction And Statistics Tool (GO-PAST); the Taxi Route Assignment and Previsualization (TRAP) tool; and the Ground-Operation Decision Support (GODS) tool. Implementation of GO-SAFE is based on objectoriented programming to enhance code maintainability, reusability, and extensibility. Figure 4 describes the high-level object-oriented architecture of GO-SAFE, where the blocks represent object classes, and the links with the diamond heads represent object aggregation or containment.

In GO-SAFE, the airport layout is based on a graphtheoretic representation, which allows the use of

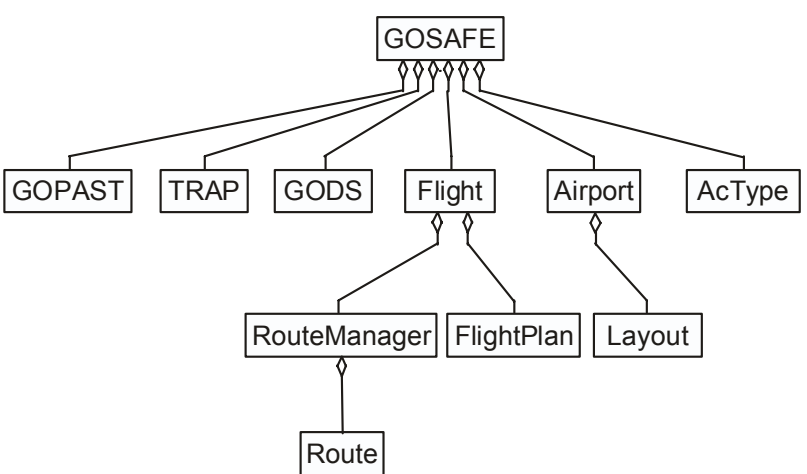

Figure 4. Object-Oriented Software Architecture of GO-SAFE

common optimization tools to compute taxi routes. The airport configurations, aircraft types, and flights are all represented as objects accessible by all the tools. The "Route Manager" allows user selection of multiple route-generation schemes, so that the tools will not be limited to the schemes already developed, assuring extensibility of the system to accommodate enhancements in the future.

The current implementation of GO-SAFE has a

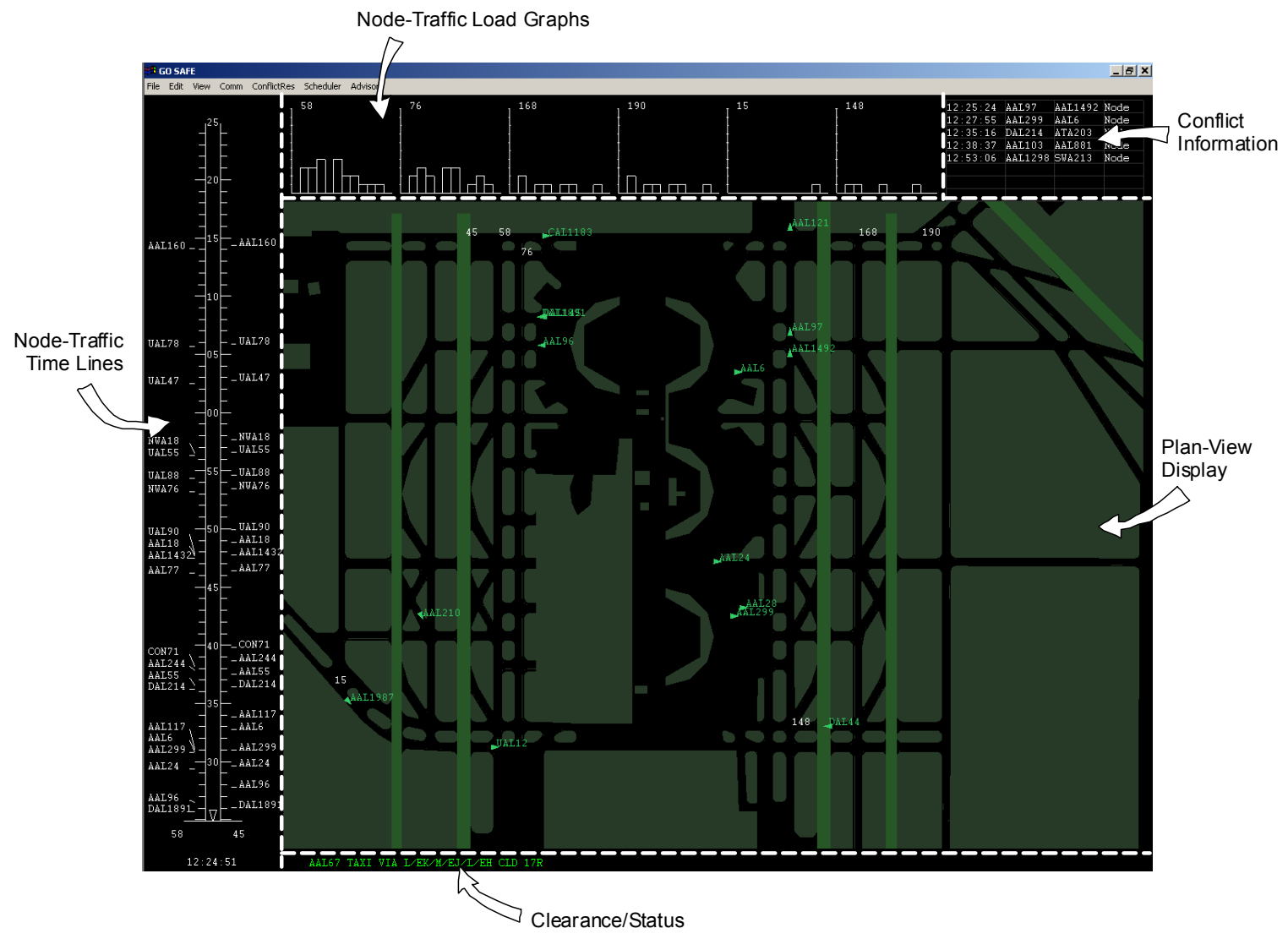

Figure 5. Overview of Experimental GO-SAFE Graphical User Interface 
graphical user interface (GUI) that includes a plan-view display for traffic monitoring and display of predicted routes. Its modularity allows replacement with new innovative user interfaces without affecting the rest of the software implementation. Figure 5 illustrates the various graphical components in this experimental GOSAFE GUI. It has five panes, the most prominent of which is the plan-view display, which shows the DFW airport layout. It shows the aircraft location based on surveillance data.

The time-line display lies to the left of the plan-view display. It shows the predicted time instants at which the flights will cross user-selected locations, which include nodes as defined by the intersections of the taxiways/runways. Above the plan-view display are traffic load graphs, which show the predicted traffic density across user-selected locations. Future enhancements may include aggregate load graphs that would provide more relevant information to the controller for predicting surface traffic congestions.

Conflict information is displayed in table form in the upper-right corner. It allows the controller to identify the conflict and resolve them manually or using the automation functions provided by GO-SAFE. The bottom of the GUI displays clearances and advisories for flights selected by the user, and the status of any issued clearances.

\section{Ground-OPERATION PREDICTION AND STATISTICS TOOL (GO-PAST)}

GO-PAST provides the functionality to allow the user to anticipate taxi traffic problems based on predicted taxi routes of the flights. The taxi routes may be initialized by GO-SAFE based on optimization and the traffic data, but they can be updated by the user through the TRAP tool, or modified by the GODS tool or other GO-SAFE functions, e.g. a conflict resolver. Currently GO-PAST provides two types of predicted traffic data: (i) density profiles of traffic passing user-specified locations as a function of time, and (ii) node-crossing traffic data indentifying flights and their crossing times for traffic passing through user-specified locations. These two types of data are displayed as density plots and node-crossing time lines as illustrated in Figure 5.

The density profile keeps track of the number of flights crossing the node as a function of time intervals. These profiles are updated at regular intervals. As illustrated in the time-line display of Figure 6, the bottom marks the current time, which is also displayed immediately below the node identifiers. The tic marks and the numeric values on the time lines indicate the minutes after the hour. As time marches forward, this time scale

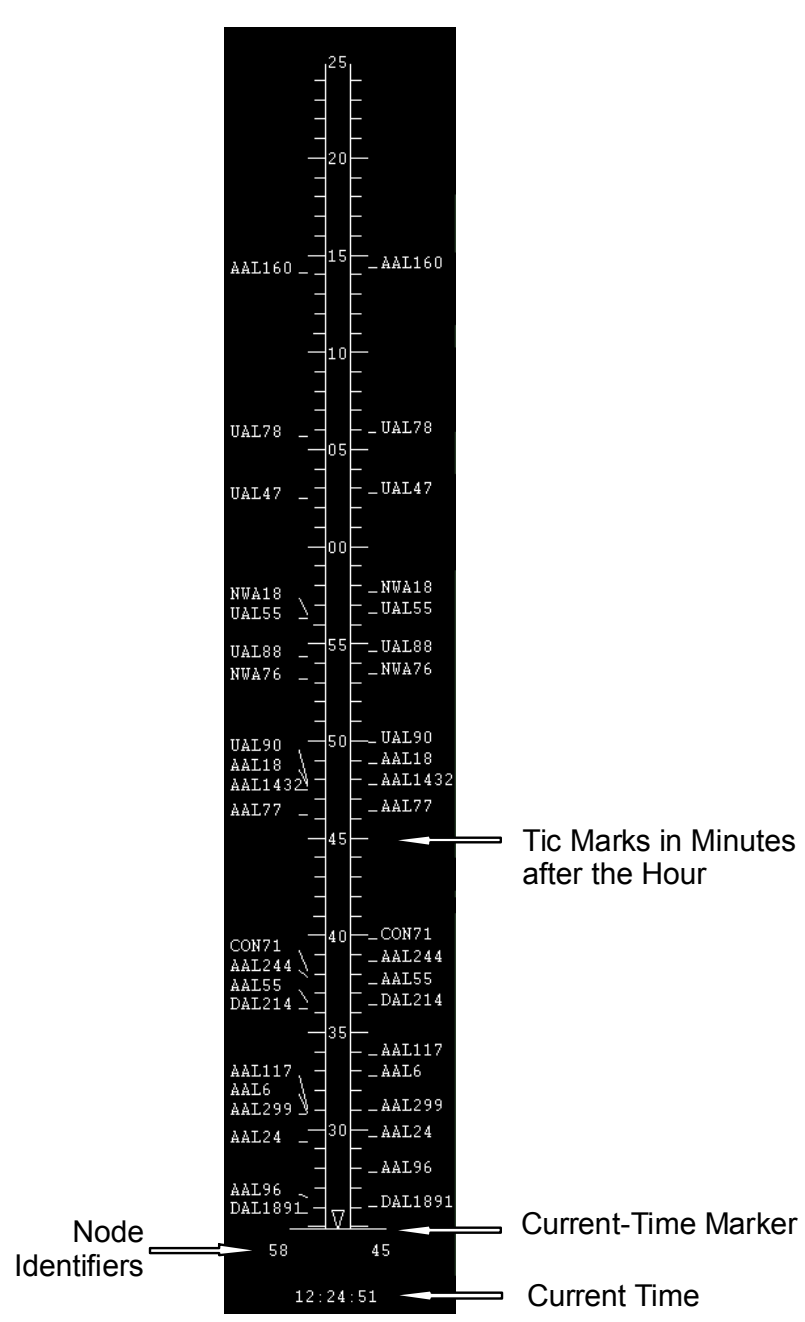

Figure 6. Time-Line Display of Node-Crossing Traffic Data

moves downward and disappears as it moves past the bottom marker.

\section{TAXI ROUTE ASSIGNMENT AND PREVISUALIZATION (TRAP) TOOL}

The TRAP tool allows the user to view taxi routes and perform manual or automatic adjustments of them. The plan-view display shown in Figure 5 provides the main viewing area for the TRAP tool. The user can select any flights to display their taxi routes and to edit them. The TRAP tool supports multiple route editing functions. In addition, information from automatic conflict detection can trigger the route editing functions to perform conflict resolution.

\section{$\underline{\text { Taxi-Route Editing }}$}

There are currently three ways to manually edit a taxi route: 
(i) modification of the final taxi location for the flight by dragging it, which is automatically followed by an automatic re-computation of the taxi route,

(ii) spatial modification of the taxi route using simple mouse clicks to define deviations from

the existing route, and

(iii) temporal adjustment of the location of an aircraft at a user-specified future time along its taxi route to control timing.

Figure 7 is an example of modifying a taxi route by dragging its final taxi location to a new location. Figure 7(a) shows the route

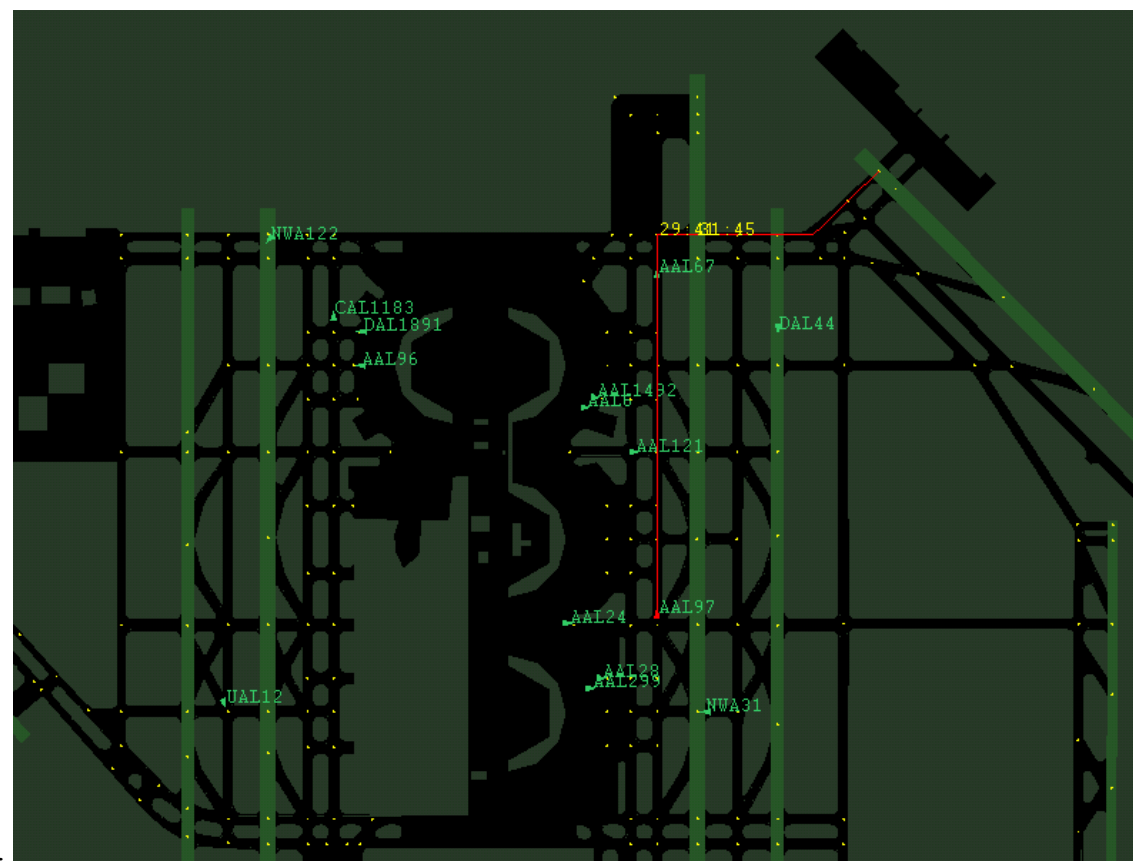

(a) Selection of a Flight Route for Editing

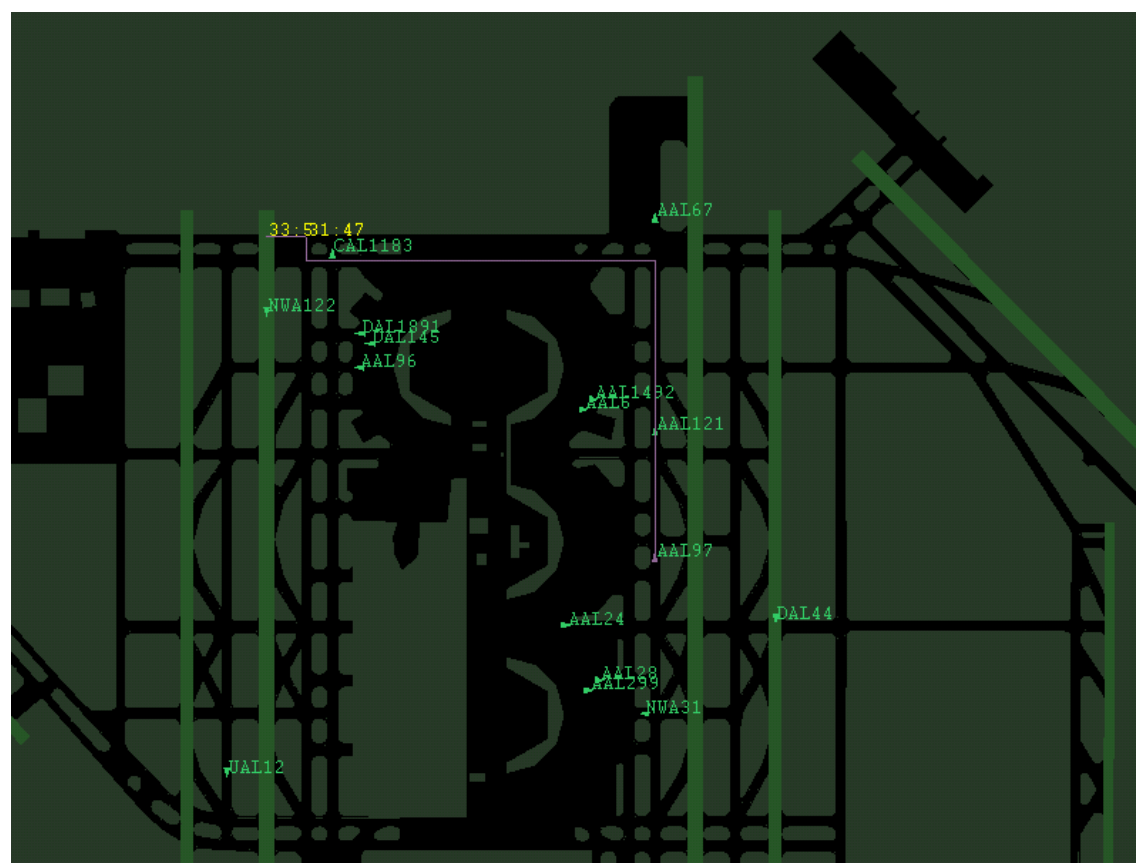

(b) Resulting Route from Dragging the Destination Node to a New Location

Figure 7. Example of Route Editing through Modification of Destination Node of flight AAL97 having been selected for editing, with taxi route ending on runway 13 . If the user wants to change the departure runway for AAL97 to $18 \mathrm{~L}$, the user can drag the destination node from runway $13 \mathrm{~L}$ to runway $18 \mathrm{~L}$. The TRAP tool will communicate to the route manager of AAL97 to request a route re-computation with the new destination node, and then it will automatically display it once it becomes available. The resulting route is shown in Figure 7(b).

Figure 8 is an example of manual modifying a taxi route using simple mouse clicks. Figure 8(a) shows that the route of flight AAL97 has been selected for editing, where the user has started defining a route deviation from the west side of runway 17R. As the user continues and ends the editing by clicking on a node along the original route, definition of the deviation is complete, resulting in the modified route of Figure 8(b). The TRAP tool checks the modified route for compliance with the airport configuration before validating it.

Figure 9 shows an example of temporally adjusting the predicted location of a flight along its defined route. Figure 9(a) shows 
the flight AAL1987 selected for viewing. To adjust its predicted position for a given time in the future, the time marker indicated by a triangular icon on the timeline display is moved to the desired time. As the time marker is dragged along the time-line scale, a square icon automatically slides along the displayed route, marking the predicted position at the specific time. With the time marker fixed at a future time, the user can drag the square icon to a new location to define it as the new location for that specific time. By defining a new future position along the route, the user is effectively imposing crossing times near that point, as indicated by the resulting display in Figure 9(b).

\section{Conflict Detection and Resolution}

The route-editing functions described in the preceding section are useful for resolving conflicts in the GOSAFE environment, either manually as they have been described, or for enabling automatic conflict resolution with further enhancements. The notion of conflicts in the GO-SAFE surface-traffic environment, however, is different from that for the air traffic, especially that under IFR. In current ground operations, the cockpit crew is responsible for aircraft separation. Consequently, the conflicts that appear in the GOSAFE route computations generally do not represent

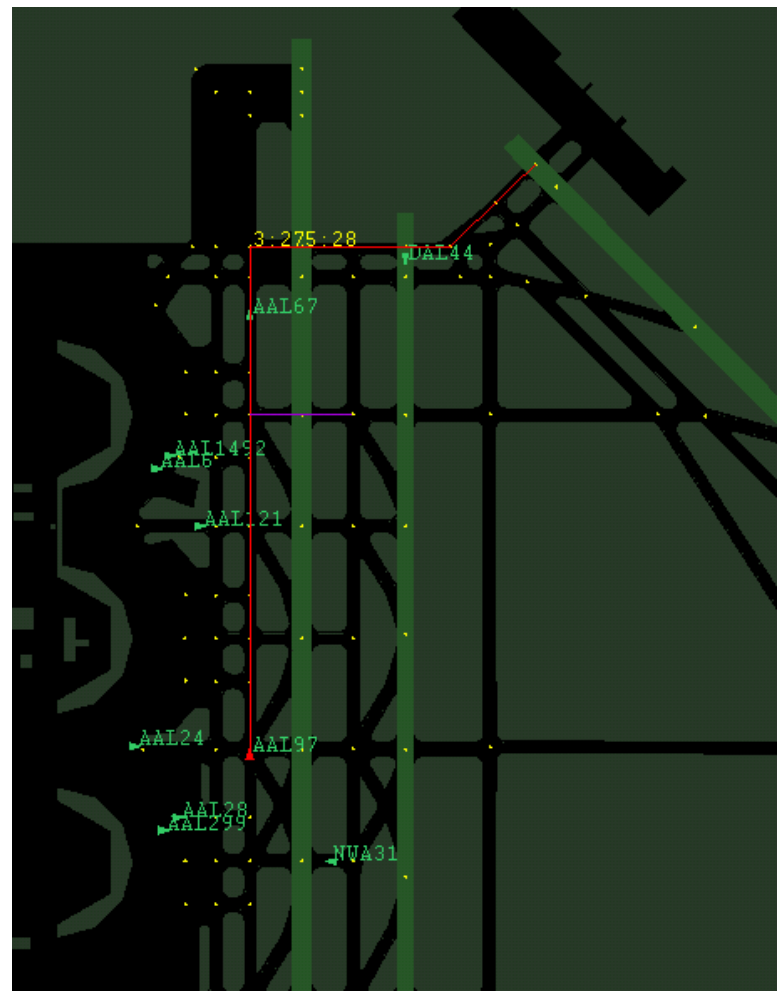

(a) Initial Route with Deviation real dangers, but rather they represent an inadequacy of the route-modeling technique based on single-vehicle consideration to accurately reflect multi-vehicle operations. For instance, two aircraft modeled in GOSAFE with one overtaking another along a taxiway does not reflect the real-world situation, where the trailing aircraft will simply follow the leading one with no attempt to pass it. It is the objective of the conflict detection and resolution functions to ensure that these situations are correctly represented in GO-SAFE, and for safety reasons to ensure that real conflicts do not happen. If future operations allow flight-deck automation for taxiing, it will be more important for GO-SAFE to ensure that all cleared taxi routes are absolutely conflict-free

Figure 10 contains an example of the conflict data on the GUI, where each row of data represents a predicted conflict, and the conflicts are ordered according to the time predicted for the conflicts to take place. Each row lists the predicted conflict time, the call sign of the two flights involved in the conflict, and whether the conflict happens at a node (i.e. taxiway/runway intersection) or an arc (i.e. taxiway/runway segment). The example in Figure 10 shows five conflicts, each of which is predicted to take place at a node.

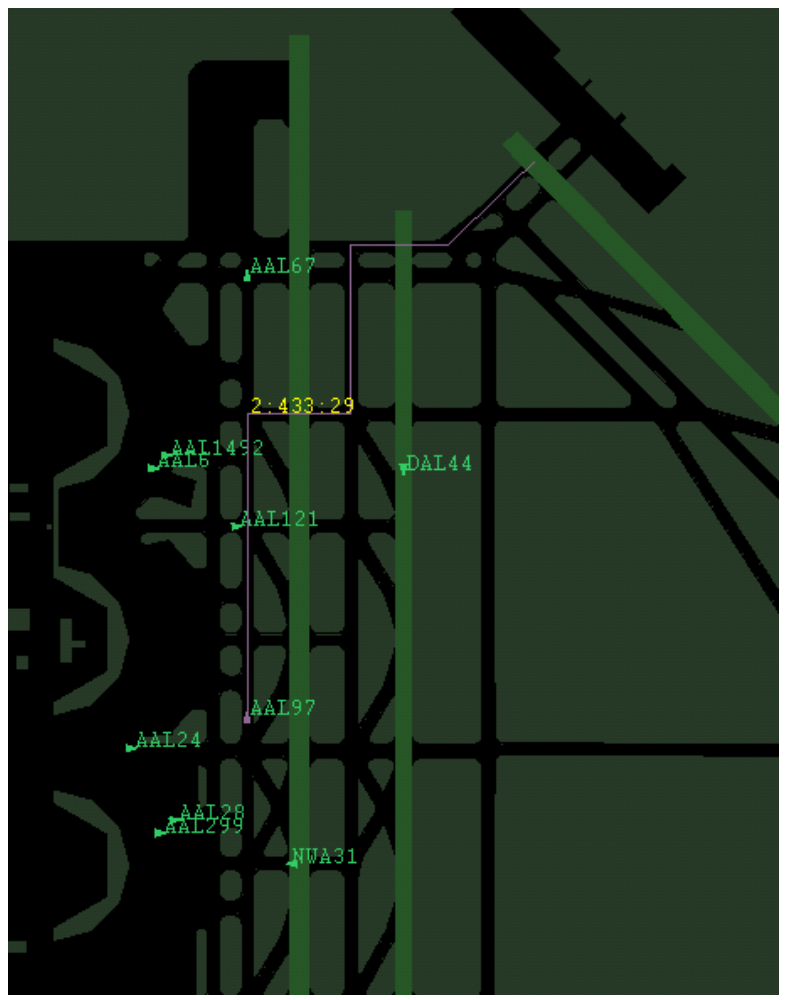

(b) Modified Route

Figure 8. Example of Route Editing through Spatial Modification 
In addition to displaying the conflict data, the TRAP tool with this display also provides capabilities for resolving the conflicts. By clicking on one of these rows, as is the case with the first row in Figure 10, the two flights involved in the conflict are automatically selected for route viewing, and the time marker is simultaneously moved (see Figure 9(a)) to the predicted conflict time. These two actions together cause the predicted locations of the two flights to be displayed. In the example here, the predicted routes of flights AAL97 and AAL1492 violate the separation requirement at an intersection, and their locations at the predicted time of conflict are depicted by the two square icons in Figure 10. With these icons, the user

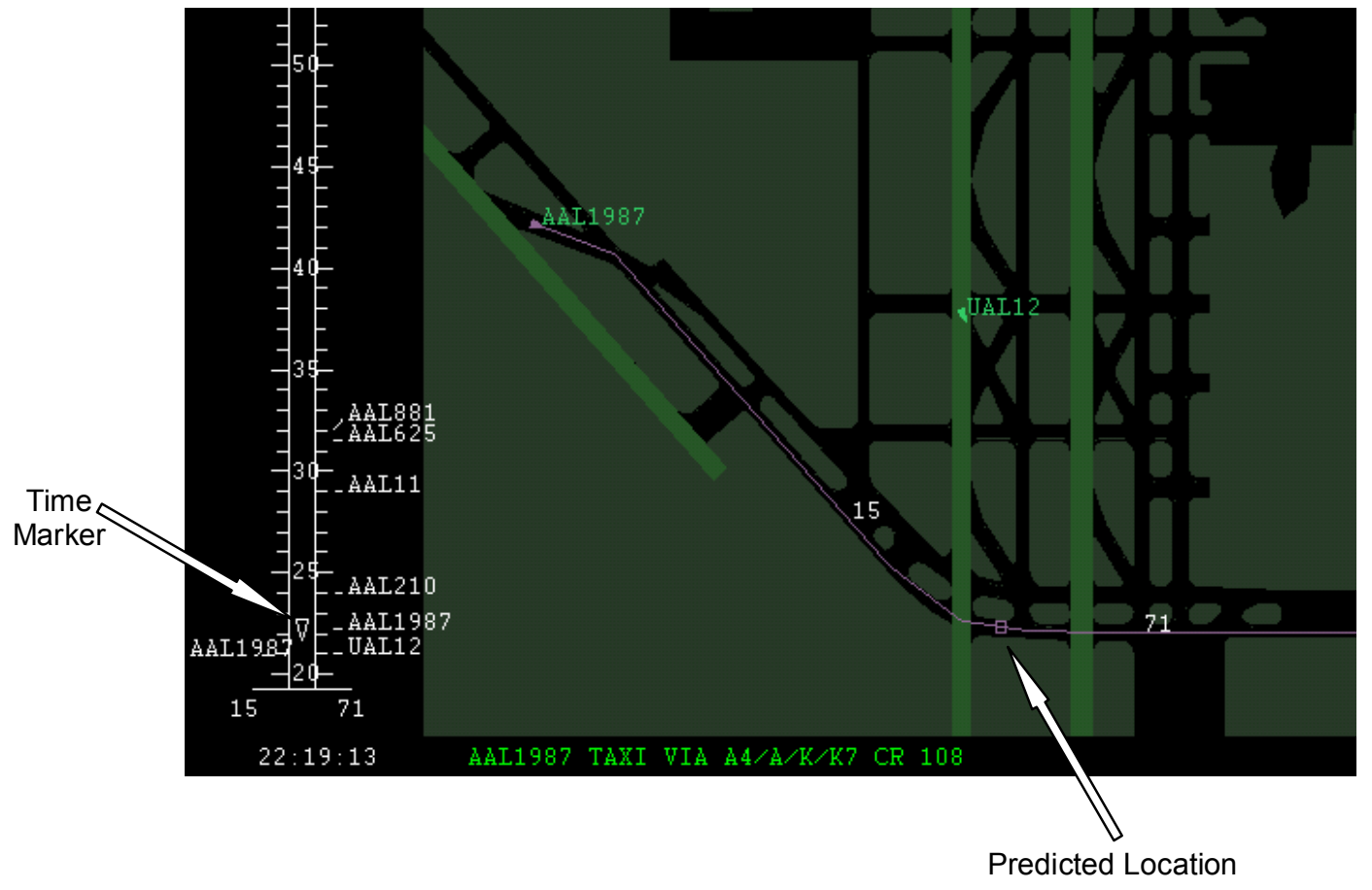

(a) Dragging Time Marker Displays Predicted Location at Selected Time

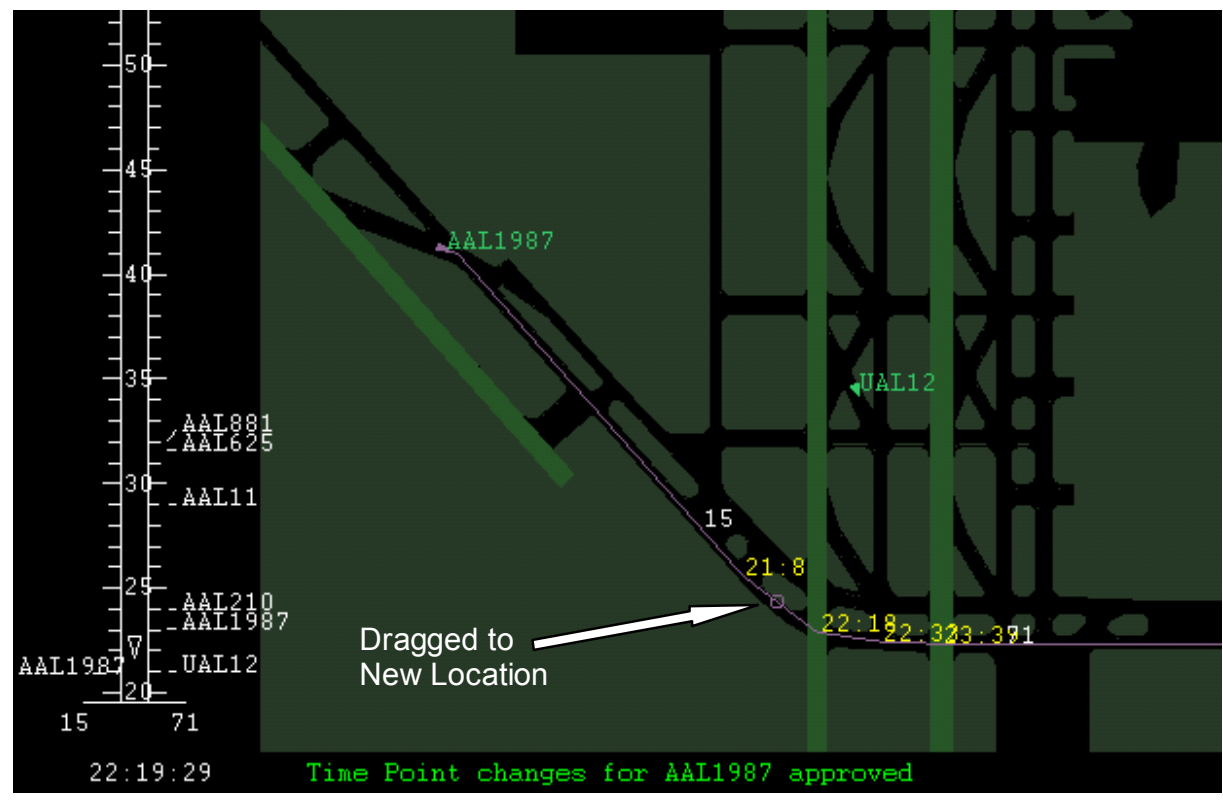

(b) Dragging Predicted Location Defines New Location for Selected Time

Figure 9. Example of Route Editing through Temporal Adjustment 
can simply drag one of them to another location along its route, thus modifying the separation at that time. Upon this change, the conflict data line will disappear, and the modified flight will have its route updated, leading to a new clearance issued with new crossing times to deliver the flight to its new predicted location at the specified time.

The TRAP tool with the conflict-data display also provides automatic methods for resolving the conflicts. If the user double-clicks on a row of conflict data, the flights in conflict are automatically selected as above, and a conflictresolution function is executed to automatically adjust the flight location to resolve the conflict. If the user prefers GO-SAFE to automatically resolve all conflicts, this option is available from the GUI menu. The conflict-resolution process can handle conflicts involving more than two flights, including the possibility of resolutioninduced conflicts.

\section{GROUND-OPERATION DECISION SUPPORT (GODS) TOOL}

The GODS tool provides decision support for ground traffic control, including issuance of advisories to the controller for possible use as clearances. It has the following main responsibilities:

- Managing and issuing advisories and clearances

- Monitoring flights to assure conformance to taxi clearances

- $\quad$ Scheduling runway usage

\section{Clearance Manager}

The clearance manager handles the advisories and clearances as dictated by the routes defined for the flights. It maintains a list of all flights with clearances that are pending (i.e. waiting to be issued), waiting for acknowledgment, or acknowledged in the negative (i.e. rejected). It is responsible for sending clearances and receiving acknowledgments. Any message that comes as part of the acknowledgment is displayed. Clearance activity is logged to assist the user in analyses of its performance. In the plan-view display, the flight icon changes color to indicate the flight's clearance status.

In addition, when a flight icon is selected, its pending or otherwise most recent clearance is displayed on the clearance/status line of the GUI (see Figure 5). If the system is under manual clearance-issuance mode, a "Send" button is available on the clearance/status line when a clearance is pending to be sent.

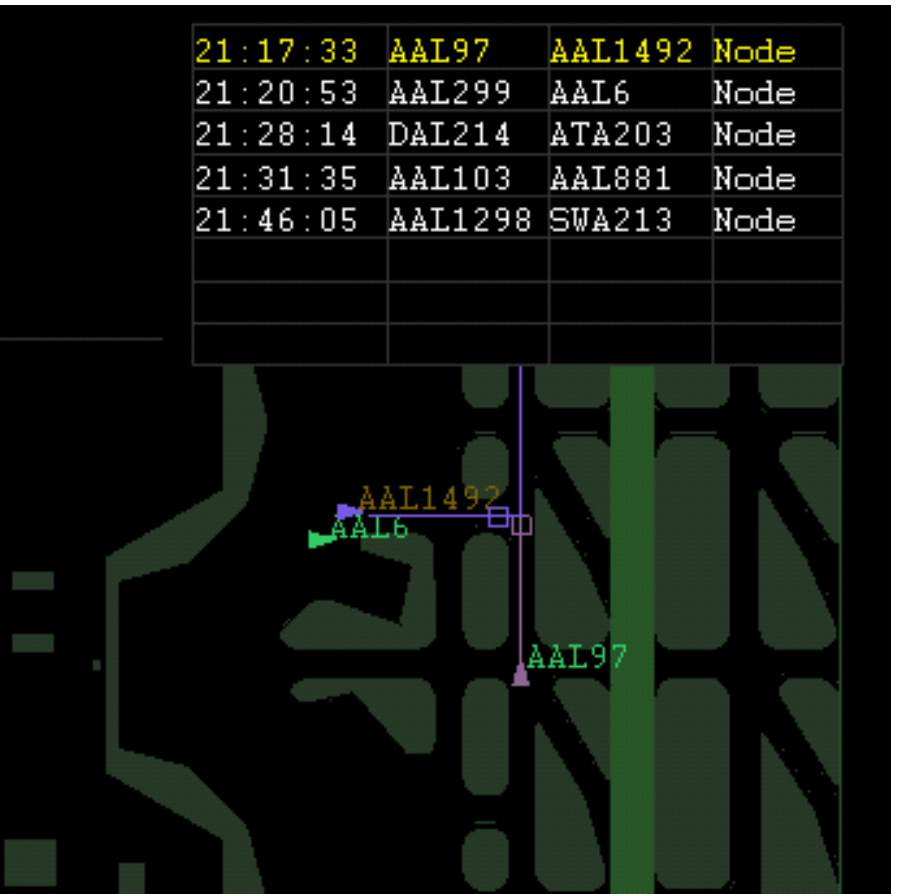

Figure 10. Traffic Conflict Data and Resolution via Manual Route Adjustment

With the taxi clearances available to the automation system, it can use the surveillance data to track the movement of the flights to assure their compliance with the issued clearances. The advantage of such automated conformance-monitoring is that it can anticipate possible locations along the taxi route where mishaps may occur. This type of capability will be highly beneficial in preventing incidents including taxi on or takeoff from the wrong path. Incidents such as these have happened in recent years in the form of unexpected takeoff from a taxiway ${ }^{21}$ or a closed runway $^{22}$.

\section{Schedule Manager}

A major objective behind the GO-SAFE development effort is the capability to allow safe and efficient activerunway crossing. To enable this capability, GO-SAFE uses a schedule manager to maintain an efficient schedule for usage of the runways by takeoff, landing and crossing traffics. The schedule manager calculates the runway-usage schedules. It contains a scheduling function that is called at regular intervals. Separate schedules are calculated for the different nodes on each runway; this approach allows for multiple aircraft crossing a runway simultaneously at different points, for example, or for an aircraft to cross the runway behind a departing aircraft. 


\section{CONCLUDING REMARKS}

Ground-Operation Situation Awareness and Flow Efficiency (GO-SAFE) represents a concept for a surface traffic automation system that builds on current and anticipated communication, navigation, and surveillance technologies to help the ground controllers at major airports move taxi traffic more efficiently and safely. It features a graph-theoretic airport-layout model to facilitate taxi-route planning. It consists of three primary tool components: the Ground-Operation Prediction And Statistics Tool (GO-PAST), the Taxi Route Assignment and Previsualization (TRAP) tool, and the Ground-Operation Decision Support (GODS) tool.

GO-PAST compiles the route data from all the flights in the system to help the controllers anticipate taxi traffic problems. It presents the data to the controller as traffic load graphs and time lines for user-selected locations. The TRAP tool provides a user-friendly graphical user interface for the controllers to examine and adjust taxi routes. Building upon the basic taxiroute editing functions, it provides additional conflict detection and resolution capabilities to enable more orderly traffic. The GODS tool provides airport-wide traffic coordination by scheduling runway usage among landing, takeoff, and crossing traffics. It provides the crucial functionality for improving active-runway crossing by accurately predicting runway-crossing windows. It also provides a clearance manager to generate advisories to the controllers, or data-linked clearances as envisioned in future ground-operation environments. Availability of the cleared taxi routes to the automation system enables the development of a conformance-monitoring function to alert the controllers or cockpit crew in case the taxi operation deviates from the cleared route.

A prototype GO-SAFE system has been developed, built around the DFW south-flow configuration. As the first prototype system, the current GO-SAFE implementation has been designed with extensibility in mind. Although its set of functions may be considered comprehensive, its object-oriented implementation allows for future additions and modifications as desirable. The experimental system has been evaluated with artificially generated traffic scenarios. Future evaluations will need to include human testing to study human-factor issues. Feedback from the research subjects can form the basis for designing new trafficcontrol schemes for integration into GO-SAFE. Furthermore, the graphical user interface has been implemented as a completely separate program from the core GO-SAFE program. This facilitates userinterface research for ground operations without the need to reprogram the core GO-SAFE functions, simply by conforming to the communications protocol.

Future research should include extensive evaluation with human subjects in a simulation environment, and extended to traffic shadowing and ultimately fieldtesting at major airports where the problem of activerunway crossing is prominent.

\section{ACKNOWLEDGMENT}

This research was supported in part by NASA under Contract No. NAS2-99055.

\section{REFERENCES}

1. V. H. L. Cheng, V. Sharma, and D. C. Foyle, "Study of Aircraft Taxi Performance for Enhancing Airport Surface Traffic Control," IEEE Transactions on Intelligent Transportation Systems, Vol. 2, No. 2, pp. 39-54, June 2001.

2. H. Erzberger, T. J. Davis, and S. Green, "Design of Center-TRACON Automation System," Proceedings of the 56th AGARD Symposium on Machine Intelligence in Air Traffic Management, Berlin, Germany, 1993, pp. 11-1-11-12.

3. T. J. Davis, K. J. Krzeczowski, and C. Bergh, "The Final Approach Spacing Tool," Proceedings of the 13th IFAC Symposium on Automatic Control in Aerospace, Palo Alto, CA, September 1994.

4. D. A. Hinton, "Aircraft Vortex Spacing System (AVOSS) Conceptual Design," NASA TM 110184, August 1995.

5. R. A. Slattery and V. H. L. Cheng, "Sensitivity of En-Route Scheduling to Variable Separation in the Terminal Area," Proceedings of the AIAA Guidance, Navigation, and Control Conference, New Orleans, LA, August 11-13, 1997, pp. 14341442.

6. D. C. Foyle, A. D. Andre, R. S. McCann, E. M. Wenzel, D. R. Begault, and V. Battiste, "Taxiway Navigation and Situation Awareness (T-NASA) System: Problem, Design Philosophy, and Description of an Integrated Display Suite for Low-Visibility Airport Surface Operations," SAE Transactions: Journal of Aerospace, 105, pp. 1411-1418, 1996.

7. R. S. McCann, D. C. Foyle, A. D. Andre, and V. Battiste, "Advanced Navigation Aids in the Flight Deck: Effects on Ground Taxi Performance Under Low-Visibility Conditions," SAE Transactions: Journal of Aerospace, 105, pp. 1419-1430, 1996. 
8. R. S. McCann, A. D. Andre, D. R. Begault, D. C. Foyle, and E. M. Wenzel, "Enhancing Taxi Performance under Low Visibility: Are Moving Maps Enough?" Proceedings of the 41st Annual Meeting of the Human Factors and Ergonomics Society (HFES), Santa Monica, CA, 1997, pp. 3741.

9. S. D. Young and D. R. Jones, "Flight Testing of an Airport Surface Guidance, Navigation, and Control System," ION National Technical Meeting, January 21-23, 1998.

10. B. J. Glass, "Automated Data Exchange and Fusion for Airport Surface Traffic Management," AIAA-97-3679.

11. D. Lawson, "Surface Movement Advisor," Journal of Air Traffic Control, pp. 25-27, January-March 1998.

12. Airport Development Plan Update - Dallas/Fort Worth International Airport, Dallas/Fort Worth International Airport Board, December 1997.

13. H. R. Idris, B. Delcaire, I. Anagnostakis, W. D. Hall, J. P. Clarke, R. J. Hansman, E. Feron, and A. R. Odoni, "Observations of Departure Processes at Logan Airport to Support the Development of Departure Planning Tools," 2nd USA/Europe Air Traffic Management $R \& D$ Seminar, Orlando, FL, December 1-4, 1998.

14. N. Pujet, B. Delcaire, and E. Feron, "Input-Output Modeling and Control of the Departure Process of Congested Airports," Proceedings of the AIAA Guidance, Navigation, and Control Conference, Portland, OR, August 9-11, 1999, pp. 1835-1852.

15. V. Capezzuto, D. Olster, M. Curry, and S. L. Pendergast, "Runway Incursion Reduction Program (RIRP) Surveillance System, NASA/FAA Atlanta Demonstration," Proceedings of the IEEE/AIAA 17th Digital Avionics Systems Conference, Bellevue, WA, Oct. 31-Nov. 7, 1998, Paper F31.

16. R. Castaldo, "Positive Identification of Aircraft on Surface Movement Areas - Results of FAA Trials," Air Traffic Control Technologies II, Orlando, FL, April 10-11, 1996, SPIE Proceedings Vol. 2737, pp. 134-142.

17. C. Evers, R. Cassel, and D. Lee, "Analysis of ADS-B, ASDE-3 and Multilaterlation Surveillance Performance - NASA Atlanta Demonstration," Proceedings of the IEEE/AIAA 17th Digital Avionics Systems Conference, Bellevue, WA, Oct. 31-Nov. 7, 1998, Paper F33.
18. V. Edwards and C. Evers, "Loop Technology (LOT) as an Alternative Surface Surveillance System," Proceedings of the IEEE/AIAA 17th Digital Avionics Systems Conference, Bellevue, WA, Oct. 31-Nov. 7, 1998, Paper F45.

19. D. S. Hicok and D. Lee, "Application of ADS-B for Airport Surface Surveillance," Proceedings of the IEEE/AIAA 17th Digital Avionics Systems Conference, Bellevue, WA, Oct. 31-Nov. 7, 1998, Paper F34.

20. Draft Manual of Advanced Surface Movement Guidance and Control Systems (A-SMGCS), ICAO All Weather Operations Panel, June 1997.

21. J. Croft, "China Airlines Take-Off Blunder Investigated," Aviation Week \& Space Technology, February 4, 2002, p. 48.

22. F. Fiorino, "SIA Flight 006 Viewed as 'Aviation System Failure'," Aviation Week \& Space Technology, May 6, 2002, p. 42-43. 